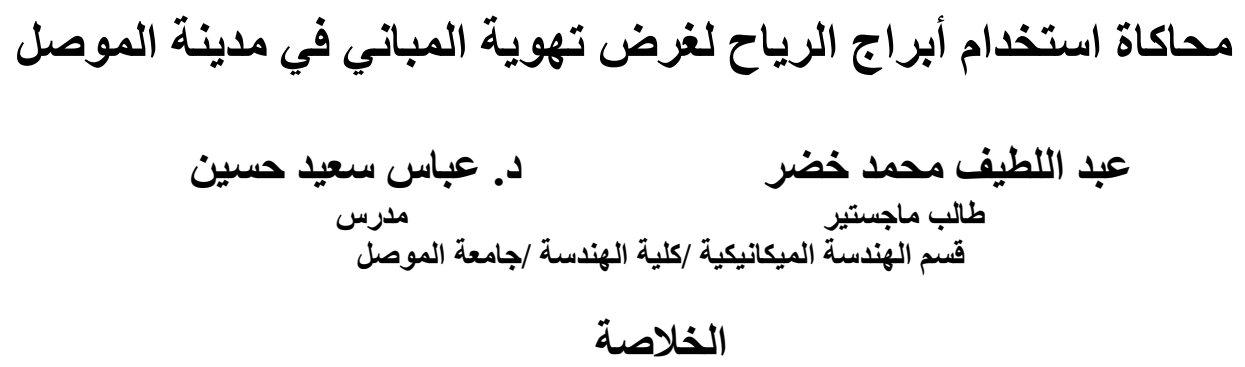

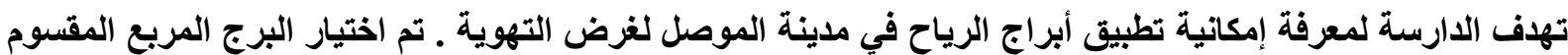

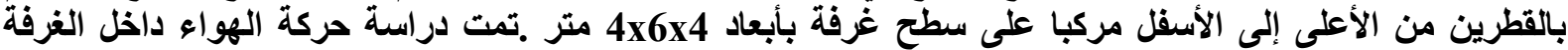

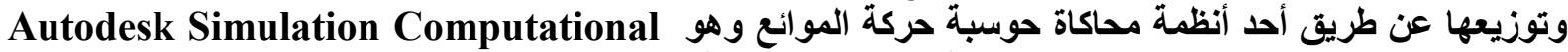

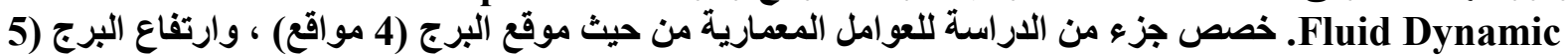

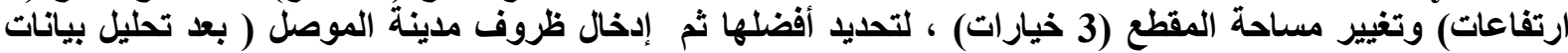

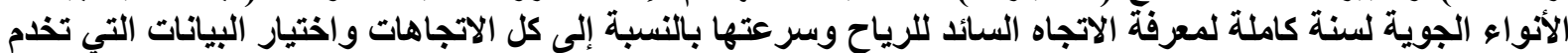

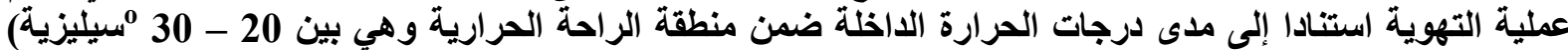

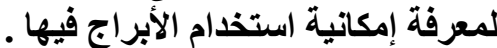

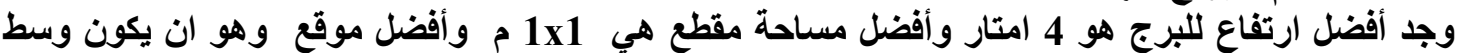

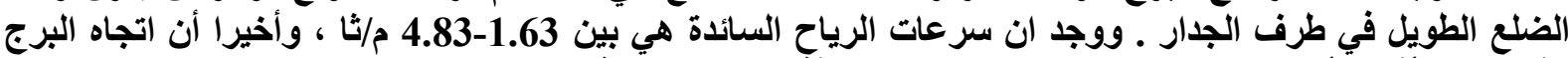

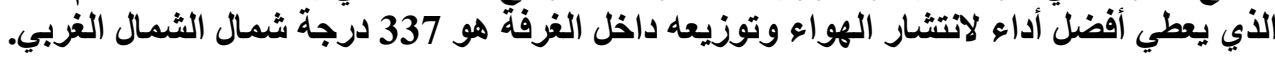

\title{
Simulation For the Buildings Ventilation using Windcatchers in Mosul
}

\author{
Abdullateef M. Khedher Dr. Abbas S. Hussein \\ University of Mosul/ Collage of Engineering/ Mechanical Eng. Dept.
}

\begin{abstract}
This research investigates windcatcher usage in Mosul for ventilation . The choice of a square shape windcatcher diagonally partitioned along its height attached to a room of dimensions 4x6x4 m. Indoor air motion speed and movement were studied to assess a windcatcher's performance .A computational model is used with Autodesk Simulation CFD software for the simulation case. Part of this study was dedicated to find the geometrical characteristics of the windcatcher parameters: - height (4 cases), crosssection ( 3 cases) and location with respect to the room (4 cases). Another part is to analyze a yearly weather data of Mosul to find the prevailing speed and direction for all cases (a range of $20-30^{\circ} \mathrm{C}$ is chosen as a thermal comfort temperature range) and to use them as an input for the simulation to find the best case of the windcatcher.

The results shows that the best heights of $4 \mathrm{~m}$, the best cross-section of $1 \times 1 \mathrm{~m}$ and the best location of the wind catcher is found to be at the middle of long side of the room. The weather data shows that the prevailing wind speed between 1.62 to $4.83 \mathrm{~m} / \mathrm{s}$. The final result of the study confirm that the direction of the windcatcher and the room which can give the best performance using all the above selected parameters in Mosul is $337^{\circ} \mathrm{NNW}$.
\end{abstract}

$$
\text { قبل: } 2015 \text { - } 4 \text { - } 28
$$$$
\text { أستلم: } 2014 \text { - } 5 \text { - } 20
$$ 
المختصرات

\begin{tabular}{|c|c|c|c|c|c|}
\hline الوحدة & التعريف & الرمز ل & الوحدة & التعريف & الرمز \\
\hline$(\mathrm{m})$ & الطول & $L$ & $\left(\mathrm{~kg} / \mathrm{m}^{3}\right)$ & كثافة الهو اء & $\rho$ \\
\hline$\left(\mathrm{N} . \mathrm{s} / \mathrm{m}^{2}\right)$ & لزوجة الاضطر اب & $\mu_{\mathrm{t}}$ & $(\mathrm{m} / \mathrm{s})$ & السر عة & $U$ \\
\hline$\left(\mathrm{m}^{2} / \mathrm{s}^{3}\right)$ & تشتت الاضطر اب & $\varepsilon$ & (N.s/m $\left.\mathrm{m}^{2}\right)$ & لزوجة الهواء & $\mu$ \\
\hline & ع-ع عامل نموذج & $\mathrm{C}_{\mu}$ & $\left(\mathrm{m}^{2} / \mathrm{s}^{2}\right)$ & للاضطر الطابة كية & $K$ \\
\hline
\end{tabular}

مقدمة

ازداد اهتمام الباحثين في العقود الأخبرة بدراسة وتطوير طر ائق التهوية الطبيعية والتر اكيب التي تخدمها وذلك التكا

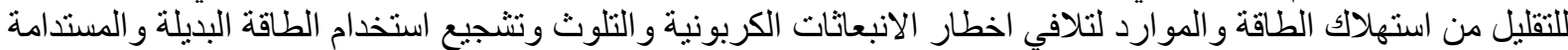

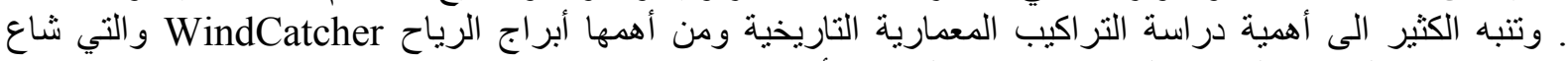

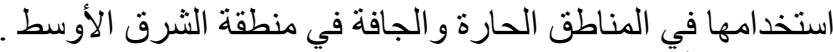

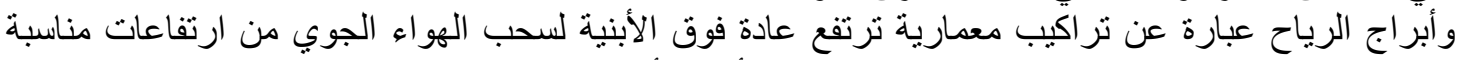

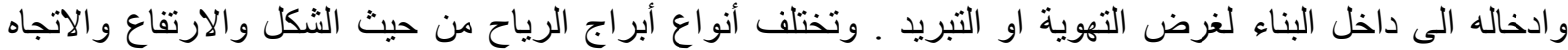

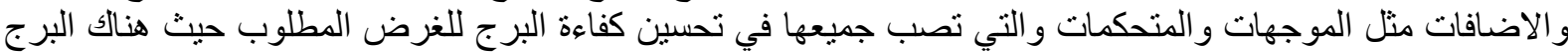

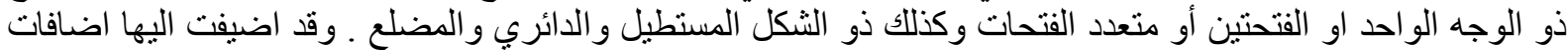

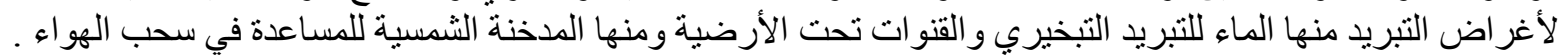

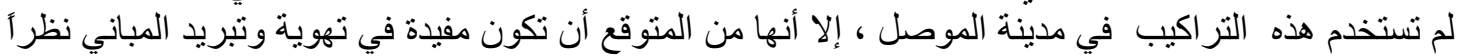

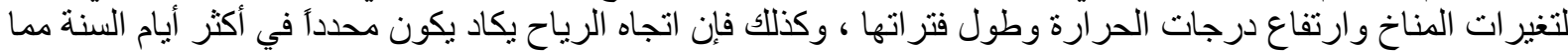

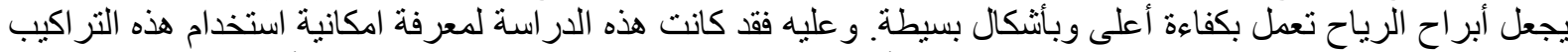

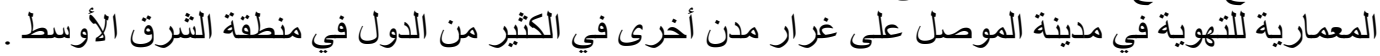

\section{البحوث السابقة}

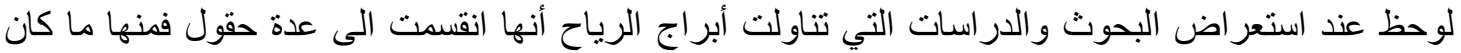

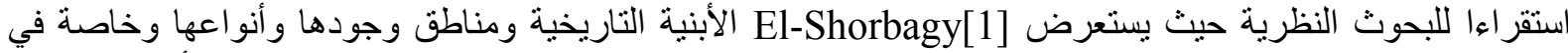

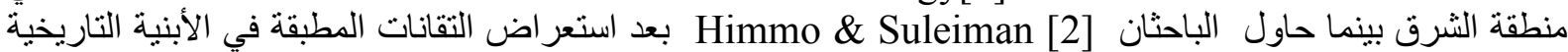

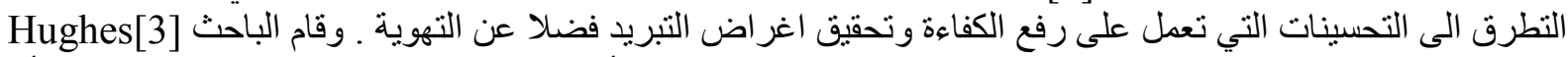

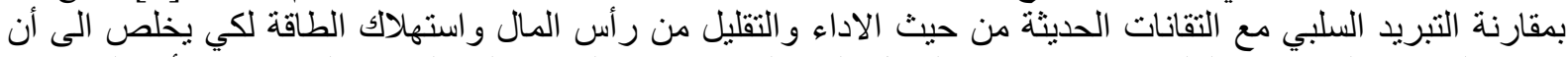

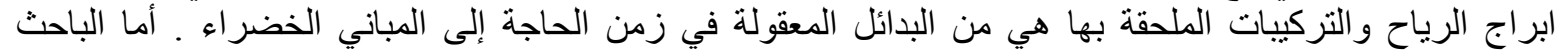

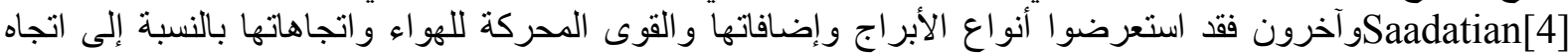

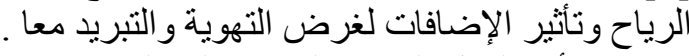

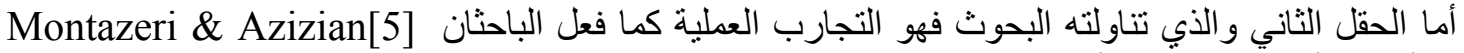

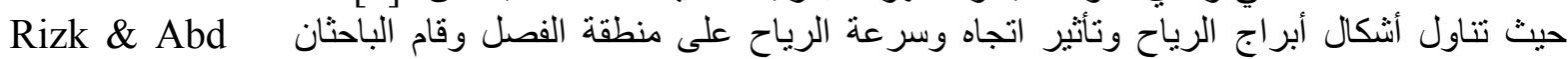

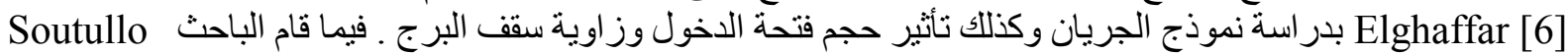

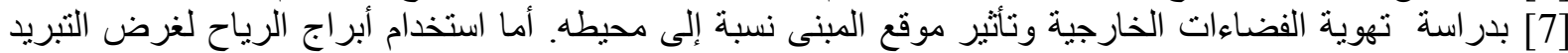

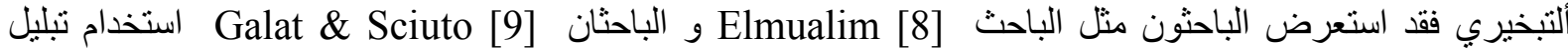

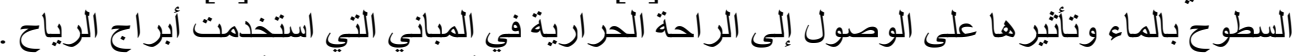

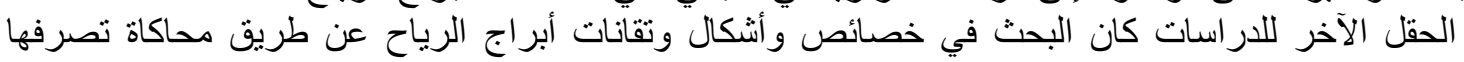

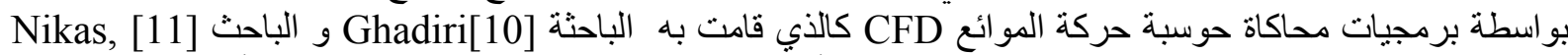

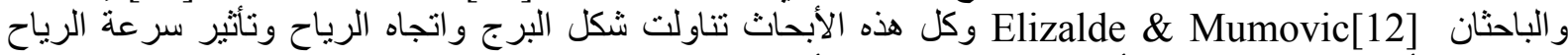

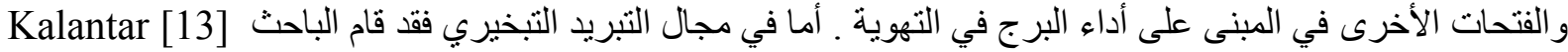

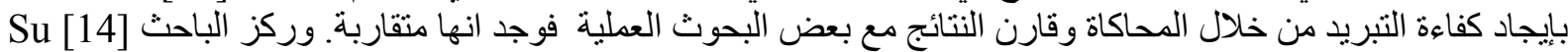

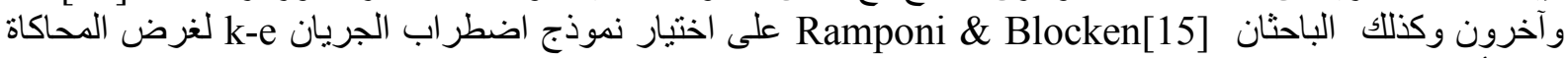
مع التأكيد على حساسية شبكة المحاكاة في النتائج . 


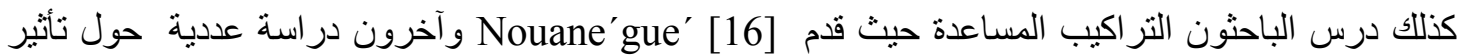

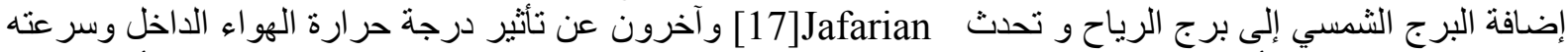

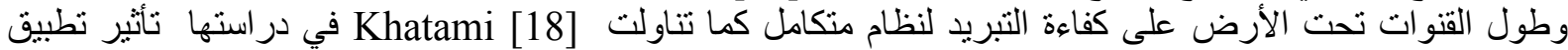

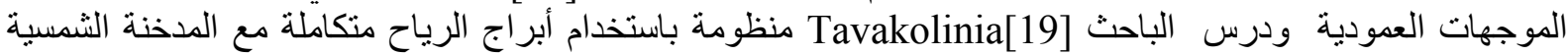

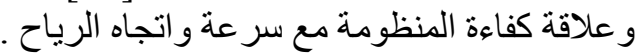

نظريـة البحث

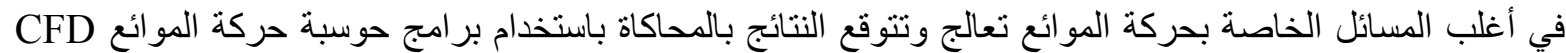

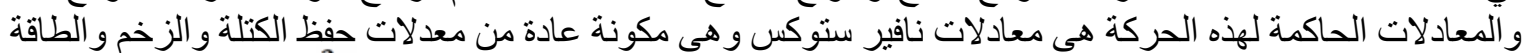

$$
\begin{aligned}
& \frac{\partial \rho}{\partial t}+\nabla \cdot(\rho V)=0
\end{aligned}
$$

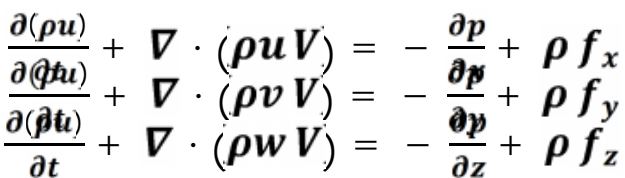

$\left.\left.\left.\left.\frac{\partial(\rho u)}{\partial t}|\rho| e+\frac{V^{2}}{2}\right)\right]+\nabla \cdot|\rho| e+\frac{V^{2}}{2}\right) V\right]=\rho q-\frac{\partial(u p)}{\partial x}-\frac{\partial(v p)}{\partial y}-\frac{\partial(w p)}{\partial z}+\rho f . v$

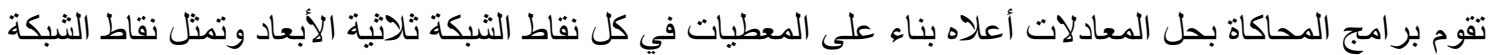

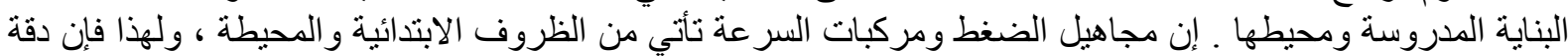

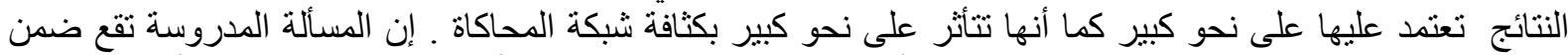
الجريان المضطرب وذللك عندما يكون رقم رينولد أكبر من

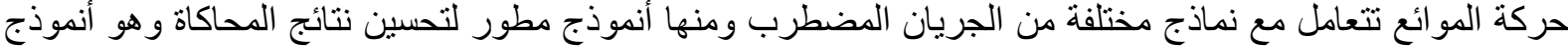

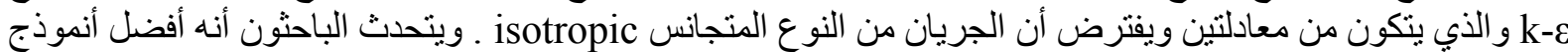

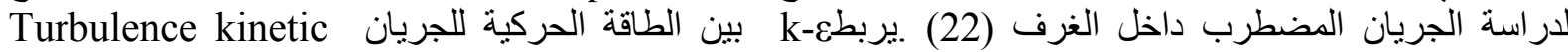

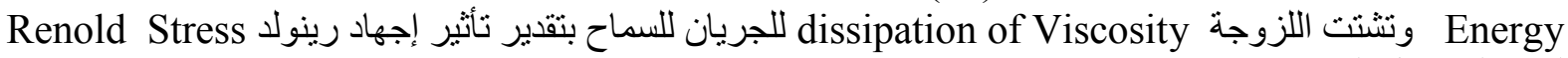

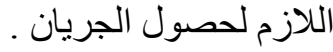

$$
0.09 \text { يفترض عادة كثابت هو }
$$

$$
\mu_{t}=\rho C_{\mu} k^{2} / \varepsilon
$$

بالمعادلة

\section{مصداقية برنامج المحاكاة}

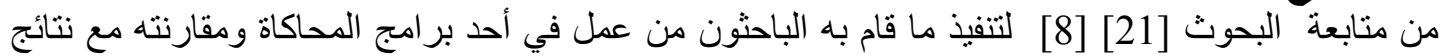

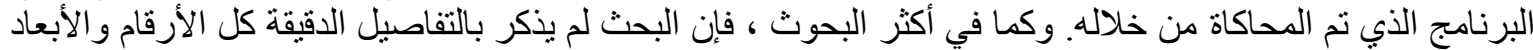

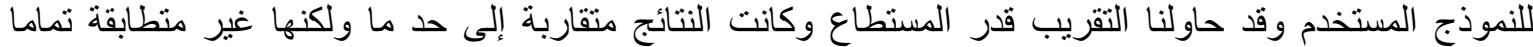

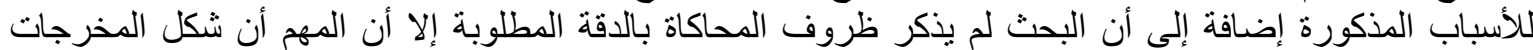

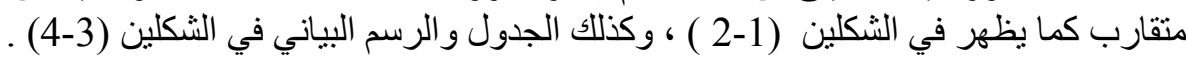
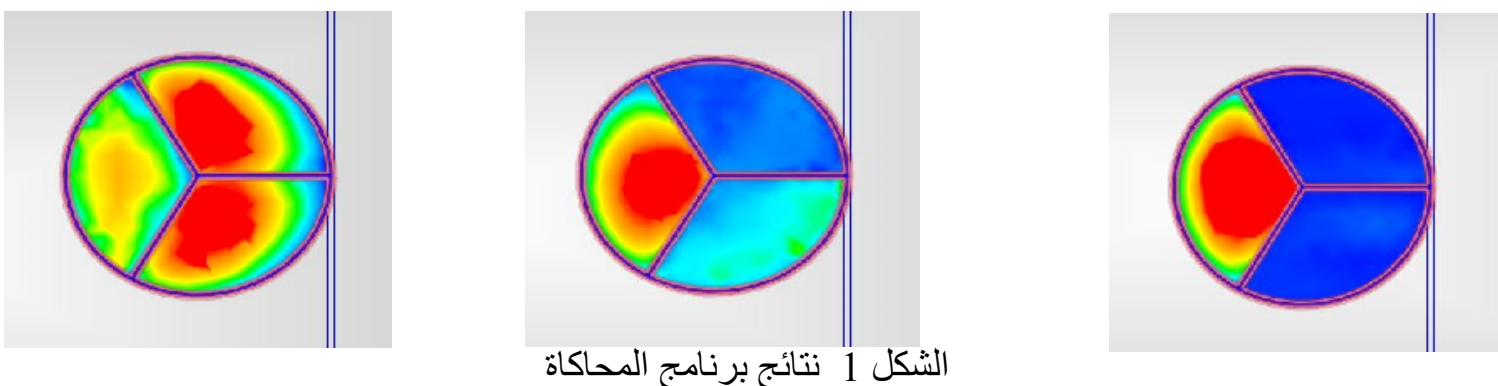

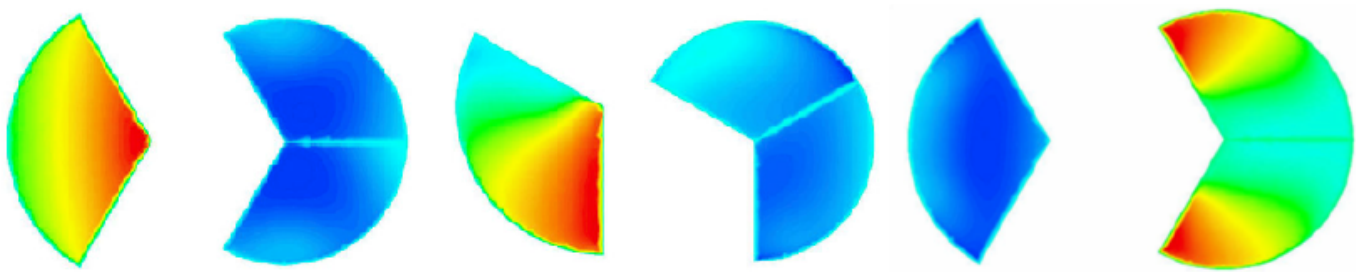

الشكل 2 نتائج البحث [21]

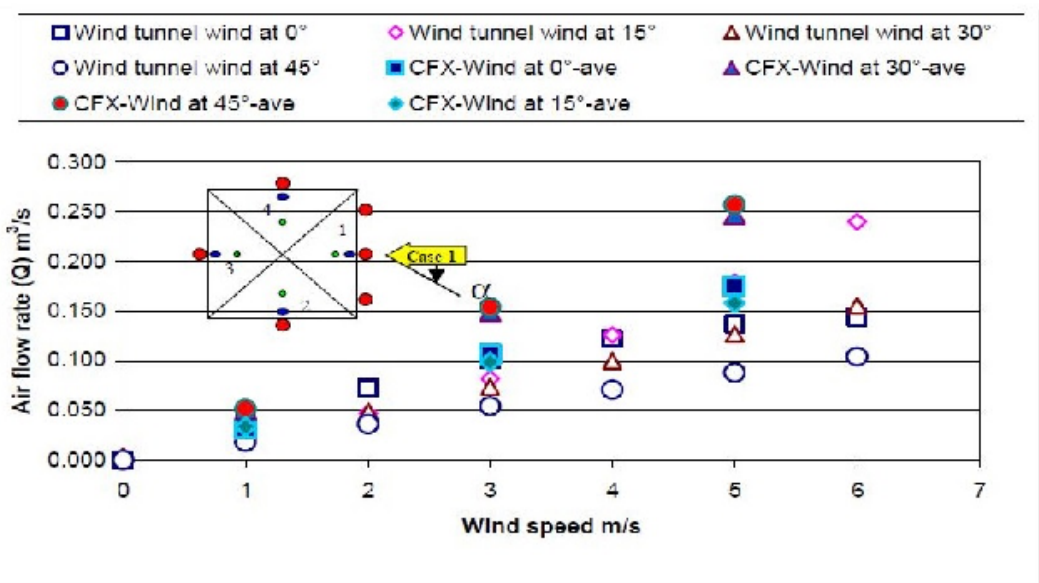

Variation of air flow with wind speed and wind direction using wind tunnel and CFD modelling

$$
\text { الثكل } 3 \text { الرسم البياني لنتائج البحث [8] }
$$

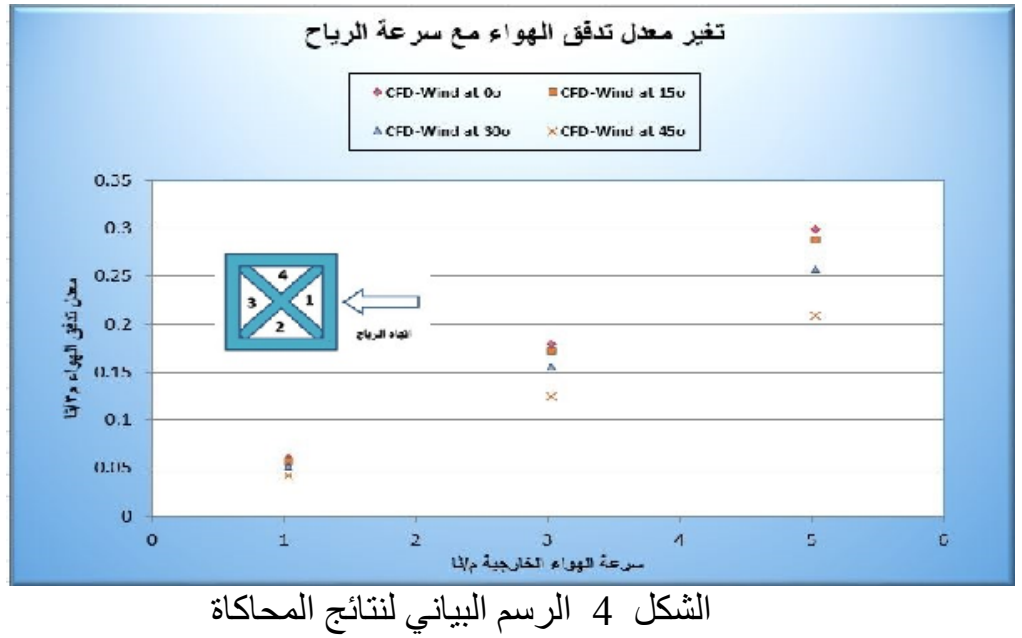

طريقة الاراسة

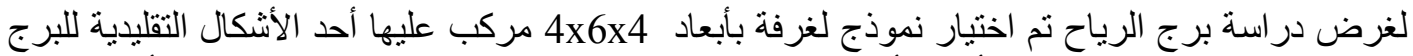

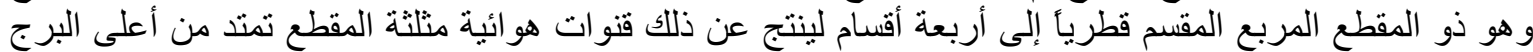

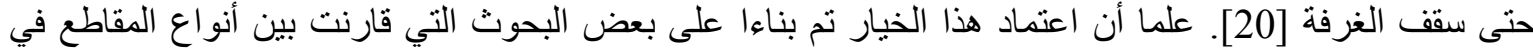

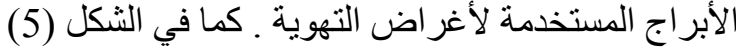



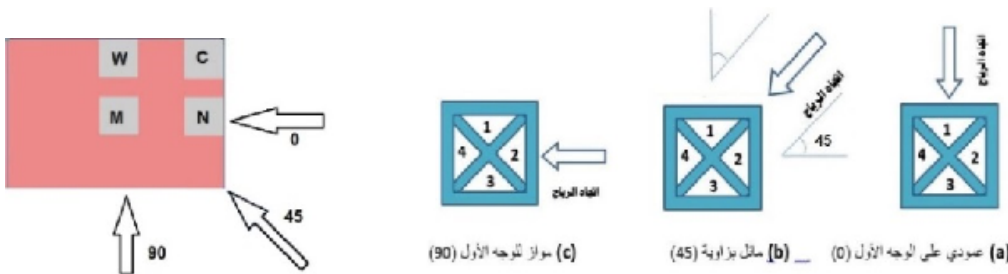

الثكل 5 شكل الغرفة النموذج وموقع و اتجاه البرج بالنسبة إلى الرياح

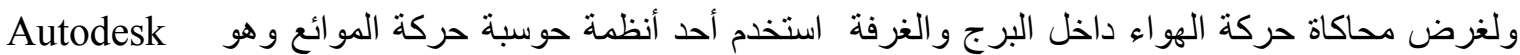

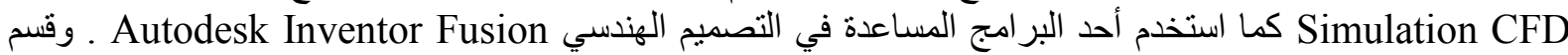

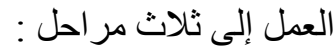

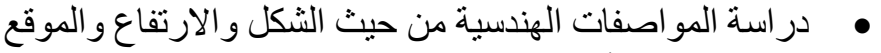

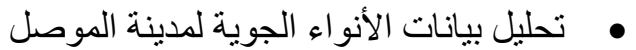

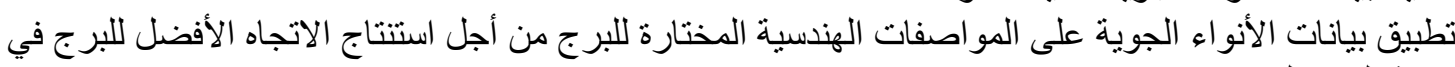

مدينة الموصل

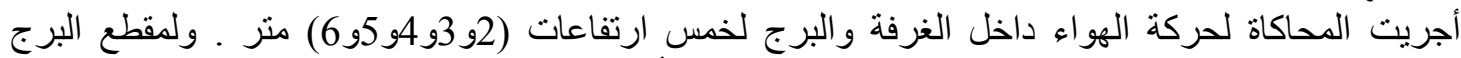

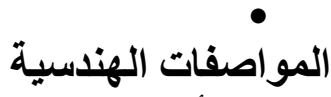

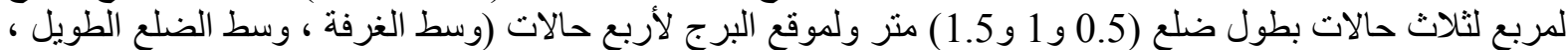

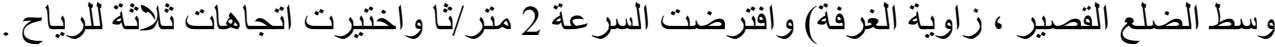

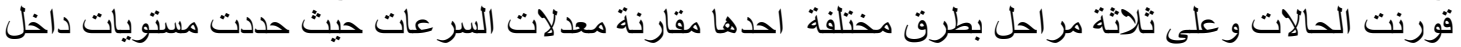

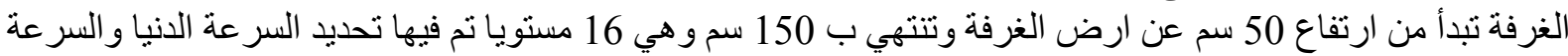

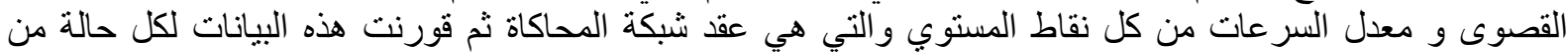
الحالات المذكورة . ولما كانت الاحتمالات كثثرة فقد نم تقسيمهاً لكي تسهل المقارنة بينها وكما في الأشكال (6-7-8)

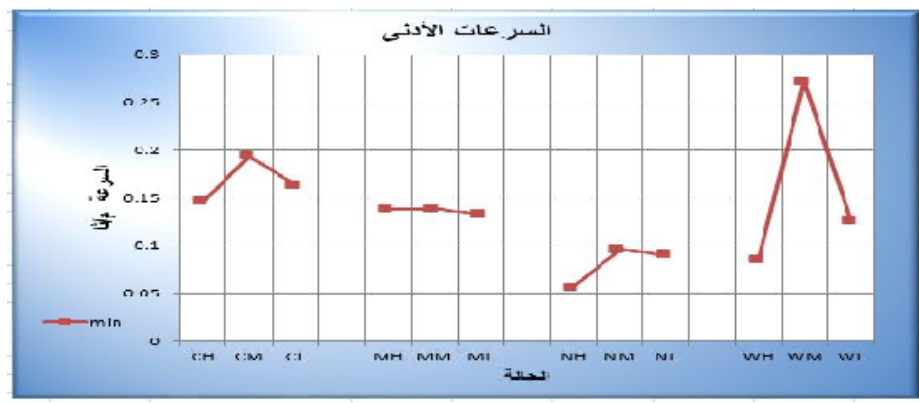

الثكل 6رسم بياني للسر عات الدنيا لجميع الحالات

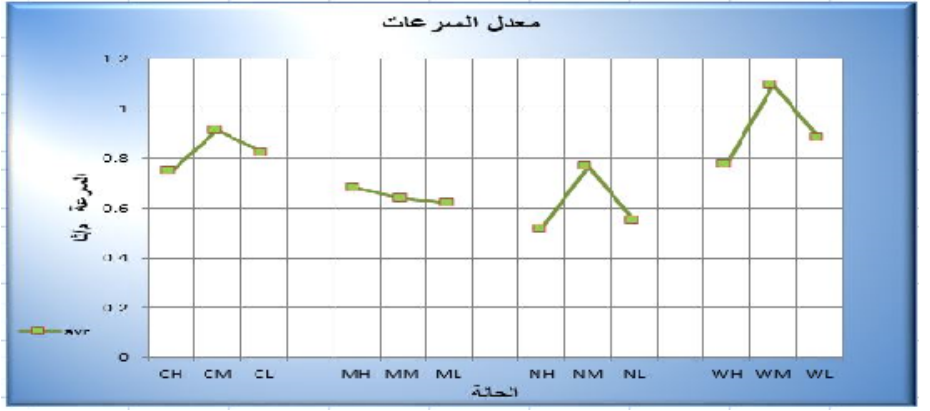

الثكل 7 رسم بياني لمعدل السر عات لجميع الحالات 


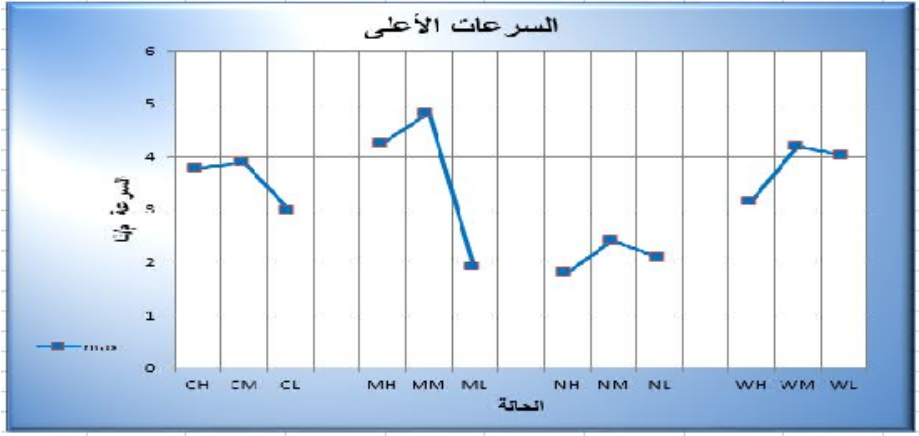

الثكل 8 رسم بياني للسر عات العليا لجميع الحالات

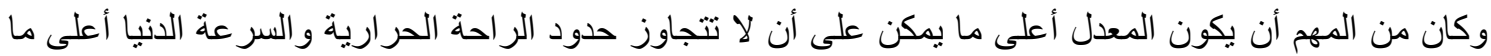

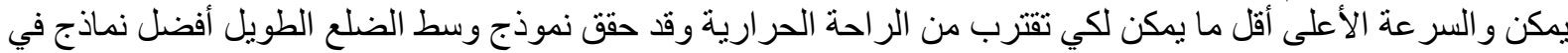

أما طريقة المقارنة الثانية فكانت عن طريق مقارنة نكر ار السر عات حيث رتبت جميع نقاط المستويات المذكورة لكل حالة

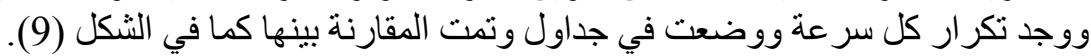

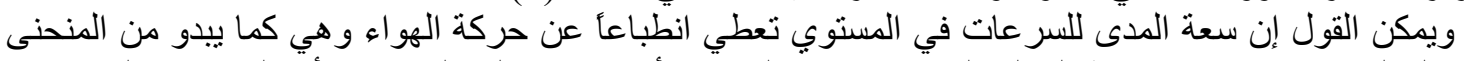

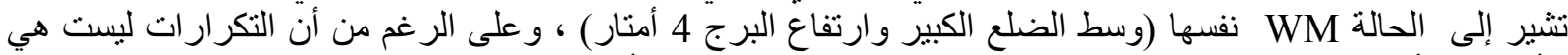

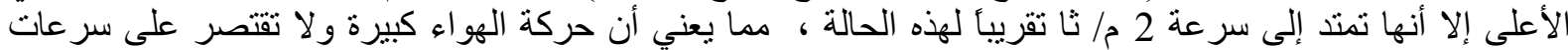

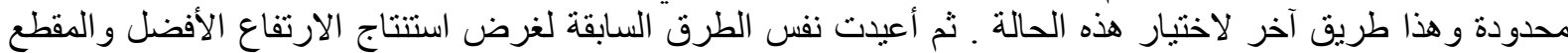

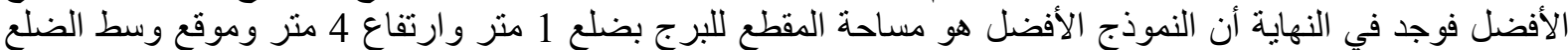
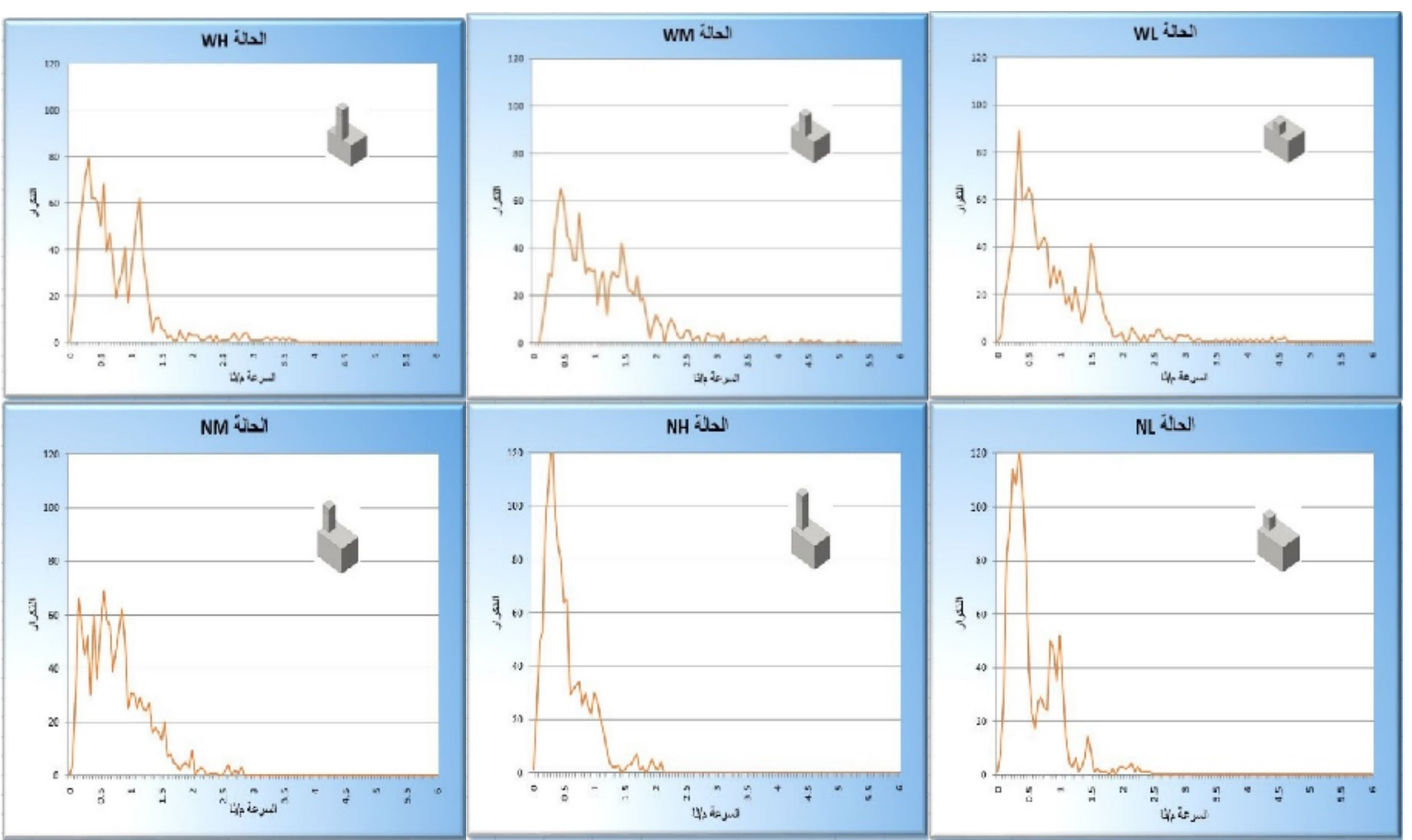
حسين: محاكاة استخذام ابراج الرياح لغرض تهوية المباني في مدينة الموصل

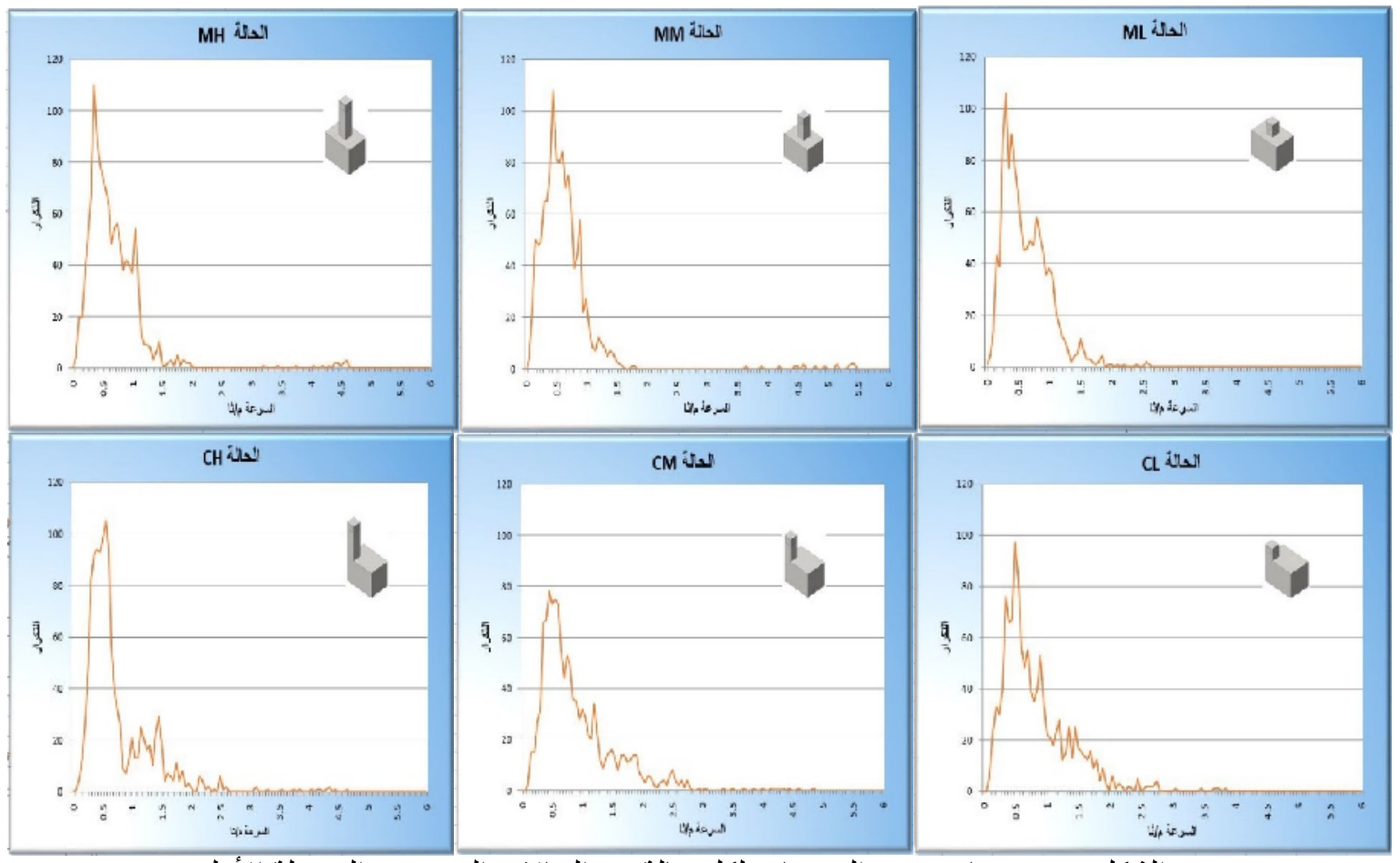

الثكل 9 رسم بياني تردد السر عات لكل حالة من الحالات الـ 12 في المرحلة الأولى

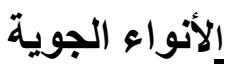

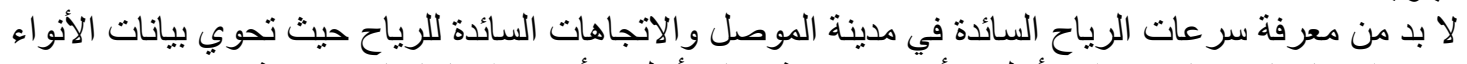

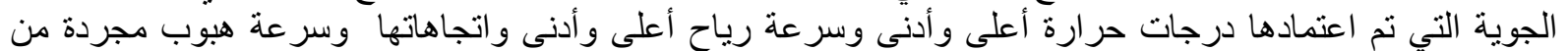

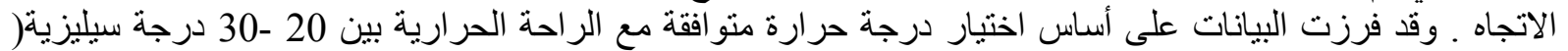
لاحظ الثكل 10) حيث أن هذه الدر اسة لا تدخل التبريد و التدفئة التي تعتبر إضافات إلى إلى أبراج الرياح .

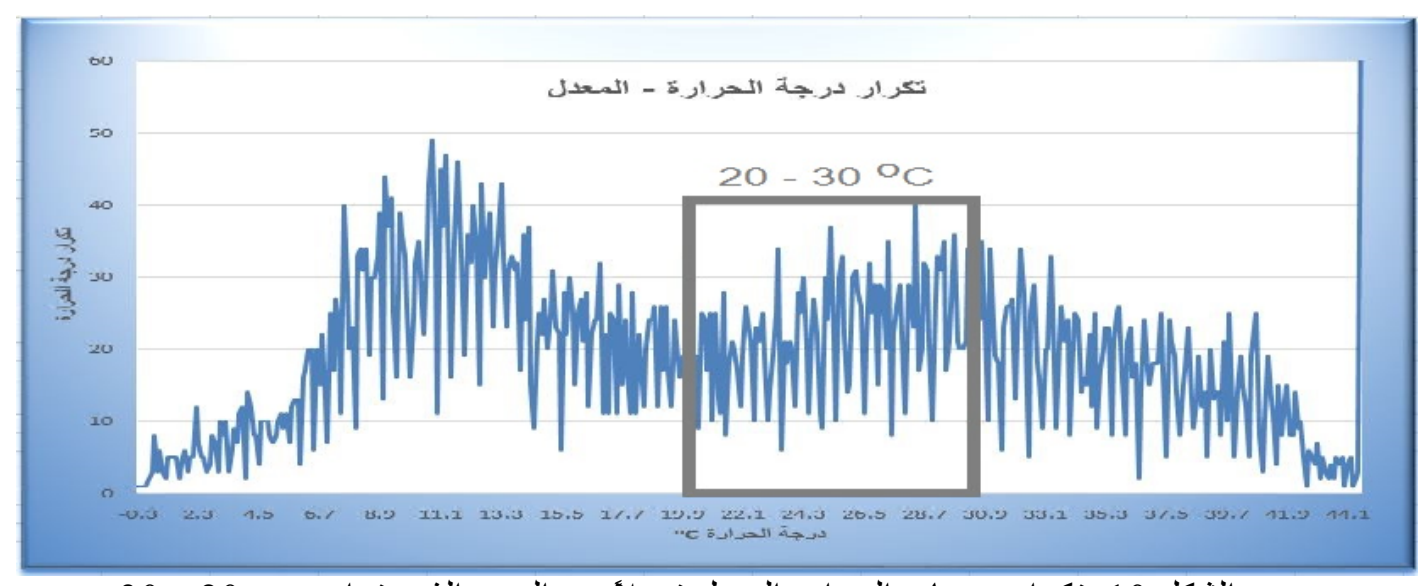

الثكل 10 نكرار درجات الحرارة المعل فضلا عن الجزء الذي يتراوح بين 20 و 30 مم

وكانت نتائج التحليل كما في الثكلين 11 و 12. 


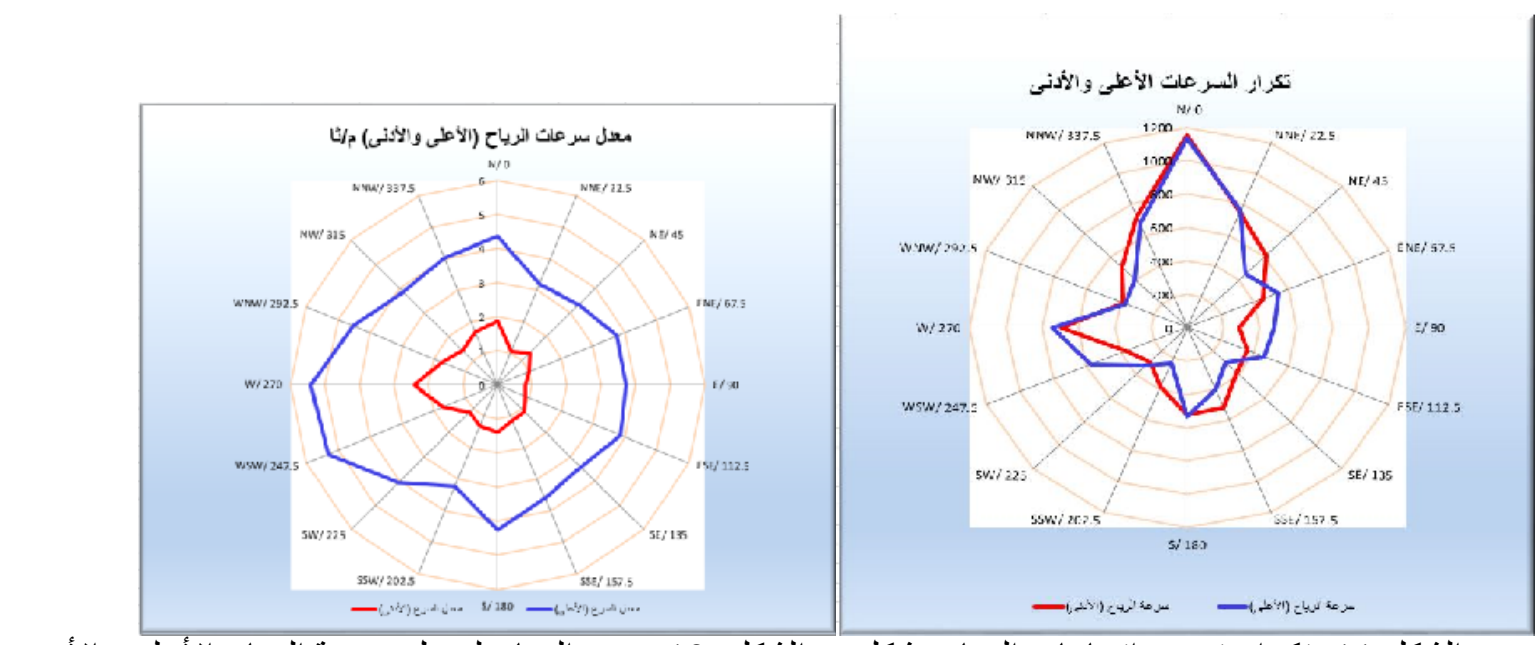

الثكل 11 نكر ار توزيع اتجاهات الرياح بشكل الثكل الثراد 12 زهرة الرياح لمعدل سرعة الرياح الأعلى و الأدنى

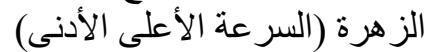

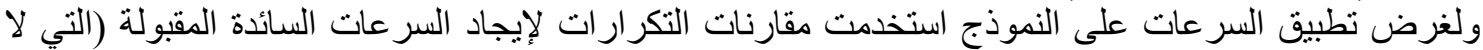

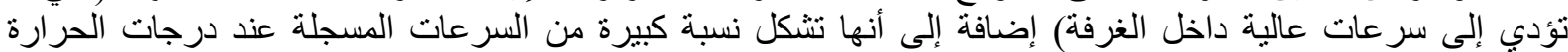

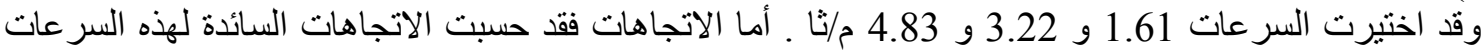

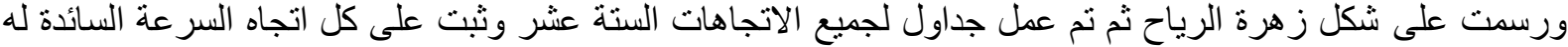

الجدول 1 السر عات المختارة والاتجاهات السائدة فيها

\begin{tabular}{|c|c|c|c|c|c|c|c|c|c|c|c|c|c|c|c|}
\hline 337.5 & 315 & 292.5 & 270 & 247. & \begin{tabular}{l|l}
5 & 225
\end{tabular} & 202.5 & 180 & 157.5 & 135 & 112.5 & 90 & 67.5 & 45 & 22.5 & 0 \\
\hline B & B & C & A & A & C & C & B & A & B & C & C & C & C & B & A \\
\hline & & \multicolumn{8}{|c|}{ الاتجاه } & \multicolumn{2}{|c|}{ السرعة } & الثرمز & & & \\
\hline & & \multicolumn{2}{|c|}{292.5} & 225 & 202.5 & 112.5 & 90 & 67.5 & 45 & \multicolumn{2}{|c|}{1.61} & C & & & \\
\hline & & & & & 337.5 & 315 & 180 & 135 & 22. & \multicolumn{2}{|c|}{3.22} & B & & & \\
\hline & & & & & & 270 & 247.5 & 157.5 & o & \multicolumn{2}{|c|}{4.86} & A & & & \\
\hline
\end{tabular}

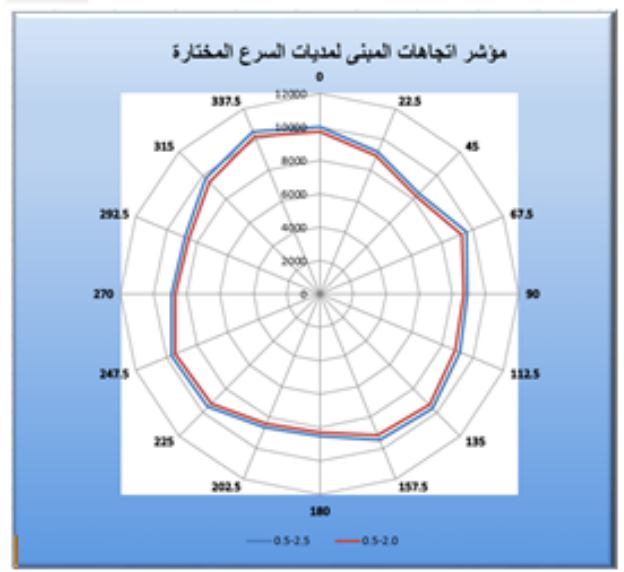

الثكل 13 ش شكل زهرة الرياح لتوضيح نأثير مديات السرعات المختارة على مؤشر الاتجاهات لتوضئ

\section{تطبيق بيانات الأنواء الجوية على النموذج المختار}

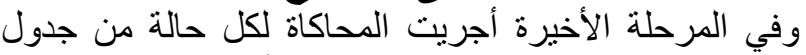

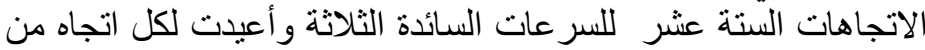

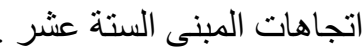
إن النتائج من المحاكاة والتي تمت مقارنتها بنفس الطرق

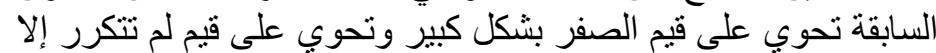

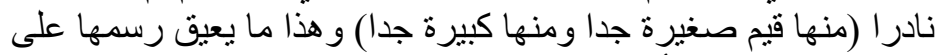

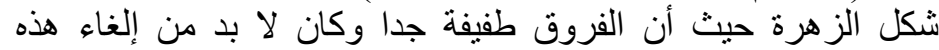
الارقام التي لا تؤثر على النتائج وقد نم اختيار المدى بن بين 0.5 و 2.0

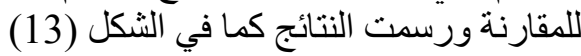

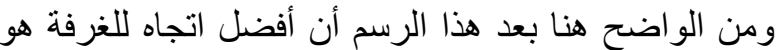
بز اوية 337.5 تقريبا أبي (شمال الثمال الغربي) كما في الثكل التالي أني

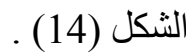




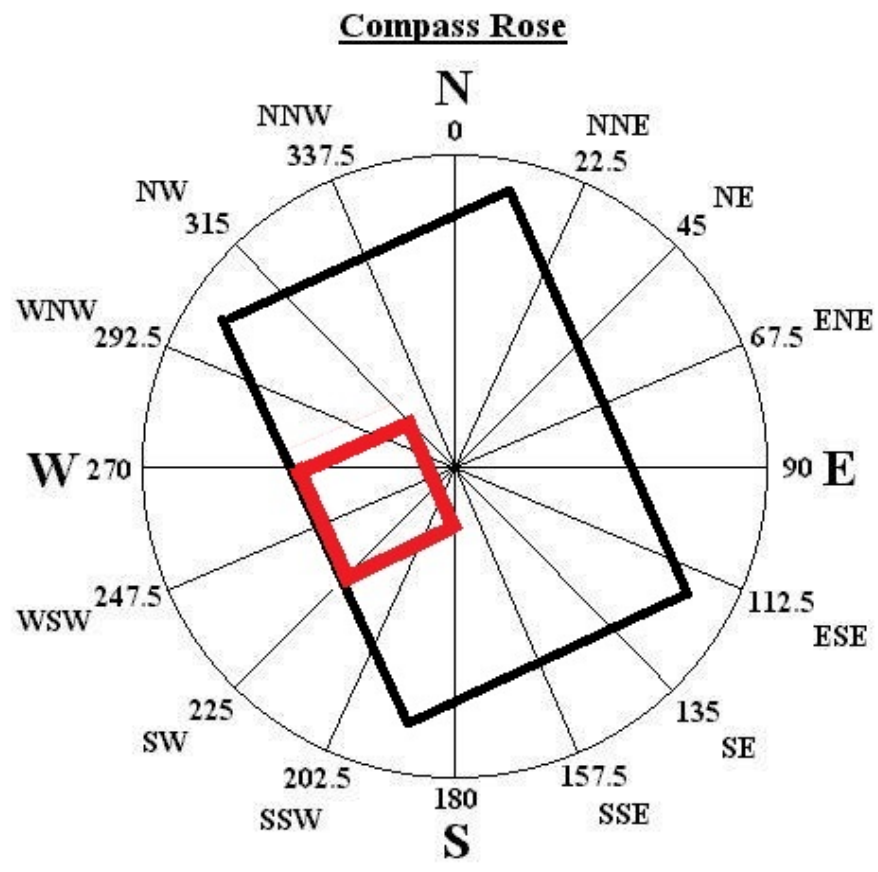

الثكل 14 رسم يوضح الاتجاه الأفضل للمبنى وفقأ لمؤشر الاتجاه في المديات المختارة من السرعة

إن التشابه بين البيانات و اضح حيث أن العلاقة الخطية متقاربة وكذلك معدلات التدفق المو افقة لسر عات الريات الرياح

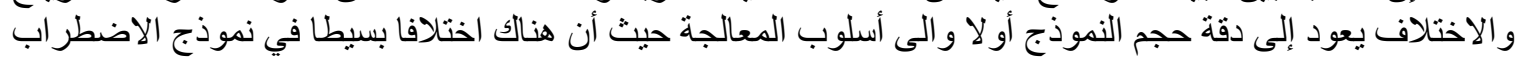
بين الحالتين .

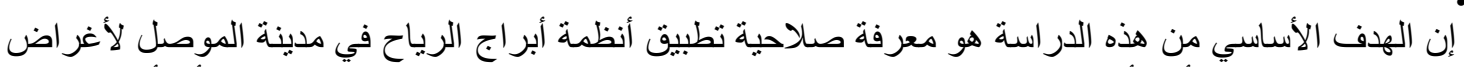

النتائج : (الن

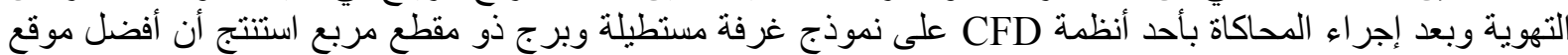

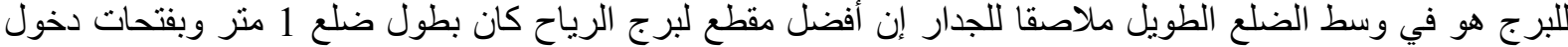

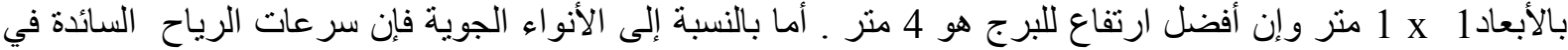

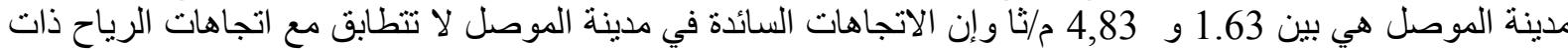

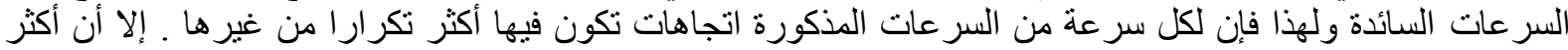

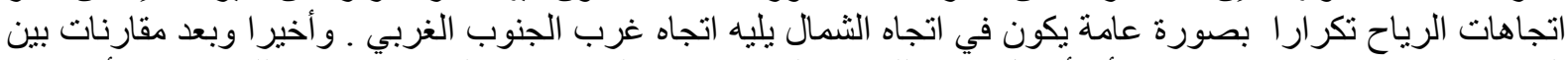

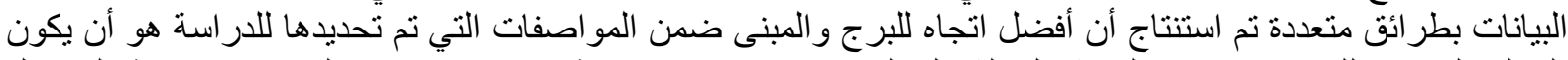

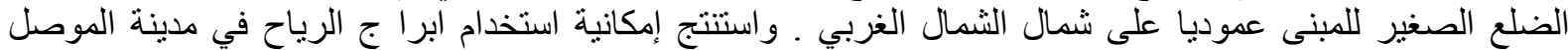

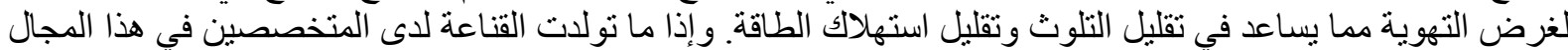

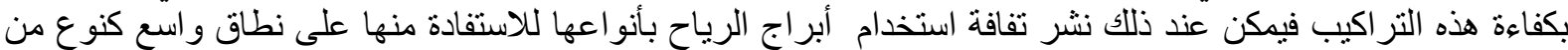

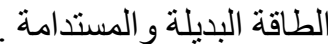

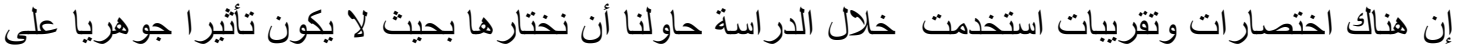

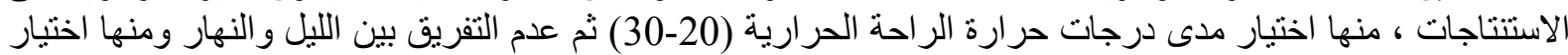

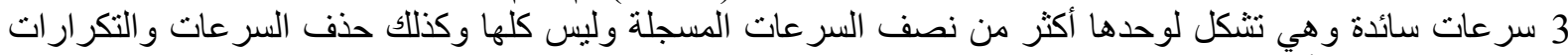

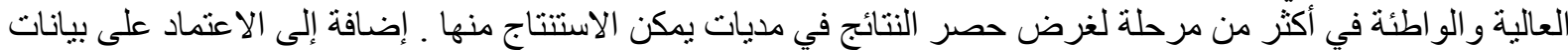

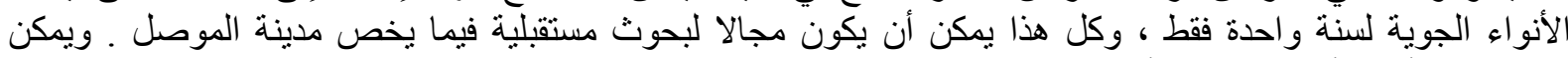

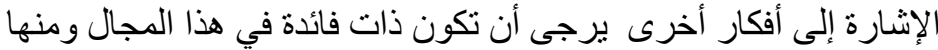


دراسة استخدام المنائر في المساجد على أنها أبراج رياح مع معالجتها معماريا من الداخل و الخارج لتحسين

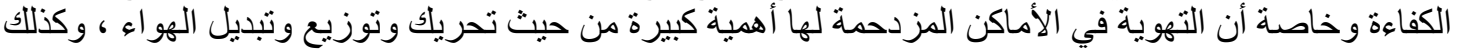

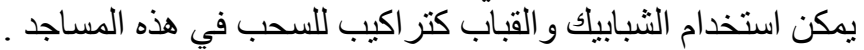

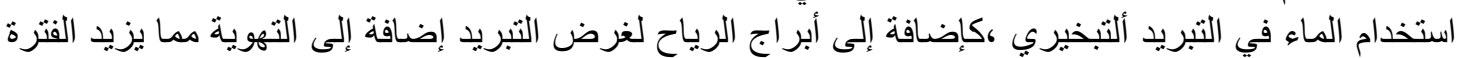

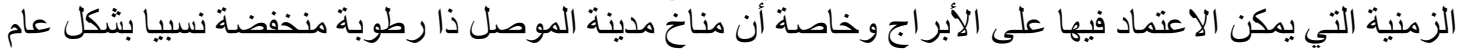

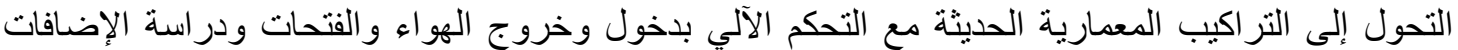

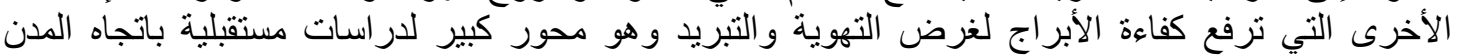
الخضر اء.

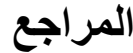

[1] Abdel-moniem El-Shorbagy "Design with Nature: Windcatcher as a Paradigm of Natural Ventilation Device in Buildings". International Journal of Civil \& Environmental Engineering IJCEE-IJENS Vol:10 No: 03, 2010.

[2] S. Suleiman, B. Himmo. "Direct comfort ventilation Wisdom of the past and technology of the future (wind-catcher)". Sustainable Cities and Society 5 (2012) 8-15

[3] Ben Richard Hughesa, Hassam Nasarullah Chaudhrya, Saud Abdul Ghanib. "A review of sustainable cooling technologies in buildings". Renewable and Sustainable Energy Reviews 15 (2011) 3112-3120

[4] Omidreza Saadatian, Lim Chin Haw, K. Sopian, M.Y. Sulaiman. " Review of windcatcher technologies". Renewable and Sustainable Energy Reviews 16 (2012) 14771495

[5] H. Montazeri, R. Azizian. "Experimental study on natural ventilation performance of onesided wind catcher". Building and Environment 43 (2008) 2193-2202

[6] Ahmed Abdel Wahab Ahmed Rizk, Mohamed Abd Elmawgoud Abd Elghaffar. "The Effect of Wincatcher -Elmalqaf- On The Internal Natural Ventilation In Hot Climate With Special Reference To Egypt. A Study On Small Physical Model". Ass. Univ. Bull. Environ. Res. Vol. 10 No. 1, March 2007

[7] S. Soutullo, R. Olmedo, M.N. Sánchez, M.R. Heras. "Thermal conditioning for urban outdoor spaces through the use of evaporative wind towers". Building and Environment 46 (2011) 2520e 2528

[8] Abbas Ali Elmualim. "Modelling of A Windcatcher for Natural Ventilation. School of Construction Management \& Engineering", The University of Reading, United Kingdom, 2004

[9] Alfio Galatà, Salvo Sciuto. "Passive Evaporative Cooling: the PDEC project". International Thermal Energy and Environment Congress (ITEEC) Marrakesh (Marocco), June 1997

[10] Maryam Hossein Ghadiri, N. Lukman N. Ibrahim, Mahmud Dehnavi. "The Effect of Tower Height in Square Plan Wind catcher on its Thermal Behavior". Australian Journal of Basic and Applied Sciences, 5(9): 381-385, 2011

[11] K.-S. Nikas, N. Nikolopoulos, A. Nikolopoulos. "Numerical study of a naturally crossventilated building". Energy and Buildings 42 (2010) 422-434

[12] Tomas Elizalde, DejanMumovic. "Simulated Performance of Windcatchers in an Urban Environment". PLEA 2008 - 25th Conference on Passive and Low Energy Architecture, Dublin, 22nd to 24th October 2008

[13] ValiKalantar. "Numerical Simulation of Cooling Performance of Wind tower (BaudGeer) in hot and arid region". Renewable Energy 34 (2009) 246-254 
[14] Yuehong Su, Saffa B. Riffat, Yen-Liang Lin, Naghman Khan." Experimental and CFD study of ventilation flow rate of a Monodraught ${ }^{\mathrm{TM}}$ windcatcher. Energy and Buildings, Volume 40, Issue 6, 2008, Pages 1110-1116

[15] R. Ramponia,b, B. Blocken. "CFD simulation of cross-ventilation for a generic isolated building: Impact of Computational parameters". Building and Environment 53 (2012) 34e48

[16] H.F. Nouane'gue' , L.R. Alandji, E. Bilgen. " Numerical study of solar-wind tower systems for ventilation of dwellings". Renewable Energy 33 (2008) 434-443

[17] S.M. Jafarian, S.M. Jaafarian, P. Haseli, M. Taheri. "Performance analysis of a passive cooling system using underground channel (Naghb)". Energy and Buildings ,Volume 42, Issue 5, May 2010, Pages 559-562

[18] Narguess Khatami. The Wind Catcher, "A Traditional Solution For Modern Problem". A Theses for the degree of Master of Philosophy, University of Glamorgan / Prifysgol Morgannwg, August 2009

[19] Fereshteh Tavakolinia. WIND-CHIMNEY Integrating the Principles of a Wind-Catcher and a Solar-Chimney to Provide Natural Ventilation. A Thesis for Master of Science in Architecture, Faculty of California Polytechnic State University, San Luis Obispo, December 2011

[20] Naghman Khan,Yuehong Su, Saffa B. Riffat. "A review on wind driven ventilation techniques. Energy and Buildings Volume 40, Issue 8, 2008, Pages 1586-1604

[21] Hamid Montazeri. "Experimental and numerical study on natural ventilation performance of various multi-opening wind catchers". Building and Environment 46 (2011) $370 \mathrm{e} 378$

[22] Mohammad Mehdi Maneshi, Amir Rezaei-Bazkiaei, A. Scott Weber, Gary F. Dargush. A "Numerical Investigation of Impact of Architectural and Climatic Parametrs of Wincatcher Systems in Induced Ventilation", Proceedings of the ASME 2012 International Mechanical Engineering Congress \& Exposition IMECE2012 November 9-15, 2012, Houston, Texas, USA 